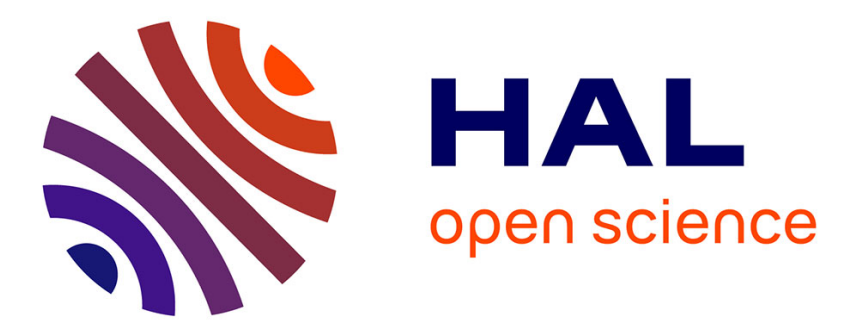

\title{
Efficacy of an oral live vaccine for veterinary use against pseudotuberculosis
}

Benoît Quintard, Thierry Petit, Nathalie Ruvoen, Elisabeth Carniel, Christian E. Demeure

\section{- To cite this version:}

Benoît Quintard, Thierry Petit, Nathalie Ruvoen, Elisabeth Carniel, Christian E. Demeure. Efficacy of an oral live vaccine for veterinary use against pseudotuberculosis. Comparative Immunology, Microbiology and Infectious Diseases, 2010, 33 (6), pp.e59-e65. 10.1016/j.cimid.2009.12.001. pasteur-02075702

\section{HAL Id: pasteur-02075702}

https://hal-pasteur.archives-ouvertes.fr/pasteur-02075702

Submitted on 21 Mar 2019

HAL is a multi-disciplinary open access archive for the deposit and dissemination of scientific research documents, whether they are published or not. The documents may come from teaching and research institutions in France or abroad, or from public or private research centers.
L'archive ouverte pluridisciplinaire HAL, est destinée au dépôt et à la diffusion de documents scientifiques de niveau recherche, publiés ou non, émanant des établissements d'enseignement et de recherche français ou étrangers, des laboratoires publics ou privés. 


\section{Efficacy of an oral live vaccine for veterinary use against pseudotuberculosis}

Benoît Quintard ${ }^{\mathrm{a}}$, Thierry Petit ${ }^{\mathrm{b}}$, Nathalie Ruvoen ${ }^{\mathrm{c}}$, Elisabeth Carniel $^{\mathrm{d}}$ and Christian E. Demeure $^{\mathrm{d} *}$

${ }^{a}$ Muséum de Besançon, La Citadelle, 25000 Besançon, France.

b Zoo de La Palmyre, 17570 Les Mathes, France.

${ }^{c}$ Ecole Nationale Vétérinaire, route Gachet, 44300 Nantes, France.

d Yersinia Research Unit, Institut Pasteur, 28 rue du Dr Roux, 75724 Paris, France.

Short Title: Live oral vaccine against pseudotuberculosis

* Corresponding author:

tel: $+33-145688448$

Fax: $+33-145688954$

christian.demeure@pasteur.fr 


\begin{abstract}
Pseudotuberculosis, an infection caused by the ubiquitous enteropathogenic bacterium Yersinia pseudotuberculosis, is a recurrent veterinary problem in livestock and zoo animals. The only vaccine currently available in zoos is Pseudovac (a mixture of killed strains of various serotypes), but its efficacy is not well established. We show here that Pseudovac does not protect guinea pigs against a severe $Y$. pseudotuberculosis infection. We thus evaluated the possibility of using a live attenuated $Y$. pseudotuberculosis strain (IP32680) as an oral vaccine against animal pseudotuberculosis. We report that IP32680 is avirulent for guinea pigs and induces a strong IgG response against various serotypes of $Y$. pseudotuberculosis. One and two oral inoculations of IP32680 provided $50 \%$ and $83 \%$ protection, respectively against a severe infection with a highly pathogenic strain. The avirulent $Y$. pseudotuberculosis IP32680 is therefore much more protective than Pseudovac and may represent a valuable oral vaccine against pseudotuberculosis in zoo animals.
\end{abstract}

Keywords: Yersinia pseudotuberculosis / pseudotuberculosis / Pseudovac / oral / vaccine / serotype / guinea pig / IgG

\title{
Résumé
}

La pseudotuberculose, causée par la bactérie enteropathogène ubiquitaire Yersinia pseudotuberculosis, est un problème récurrent pour les animaux d'élevage ou sauvages en captivité. Pseudovac, un vaccin composé de bactéries tuées de différents sérotypes, est actuellement utilisé dans les zoos bien qu'il n'ait pas été formellement testé. Dans le présent travail, Pseudovac n'a pas conféré de protection à des cobayes, une espèce très sensible, contre des doses élevées d'une souche virulente de Y. pseudotuberculosis. La souche IP32680 de Y. pseudotuberculosis, avirulente chez la souris, a été évaluée comme vaccin oral. Nous rapportons que IP32680 est avirulente pour le cobaye et induit de forts taux d'IgG contre les principaux sérotypes de $Y$. pseudotuberculosis. La vaccination par une ou deux inoculations orales de IP32680 a protégé respectivement 50 et $83 \%$ des animaux. Ce vaccin vivant oral est donc beaucoup plus protecteur que Pseudovac et peut donc représenter une alternative utile en médecine vétérinaire.

Mots clés: Yersinia pseudotuberculosis / pseudotuberculose / Pseudovac / oral / vaccin / sérotype / cobaye / IgG 


\section{Introduction}

The Gram-negative enteropathogenic bacterium Yersinia pseudotuberculosis is widely distributed in countries with temperate or cold climate and human cases of pseudotuberculosis are commonly reported in Europe, North America, Japan and Eastern Russia [1]. Such cases are most often sporadic, although outbreaks have been reported in Japan, Finland and Russia [2-4]. Ubiquitous in the environment, Y. pseudotuberculosis is usually contracted primarily through the consumption of contaminated water or greeneries. Y. pseudotuberculosis is enzootic in various mammalian and bird species, and rodents are the main reservoir of infection [5]. Contamination of livestock animals such as cattle [6], buffaloes [7], deers [8] and sheep [9] with $Y$. pseudotuberculosis also occurs and has a significant economic impact. Pseudotuberculosis is also a recurrent health problem for precious animals in zoological gardens [10-13] and wildlife parks [14] where the density of populations is high. This infection may thus be of major concern for the preservation of endangered species.

The bacterial cells absorbed orally disseminate from the gastrointestinal tract to the mesenteric lymph nodes, causing diarrhea, abdominal pain and fever. In weakened individuals, bacteria disseminate to visceral sites such as the spleen and liver, and septicemia is often observed in humans [15] and animals. Infection is treated by means of antibiotherapy, however some $Y$. pseudotuberculosis strains naturally resistant to several antibiotics have recently been observed ([16] and Rosso ML. et al., manuscript in preparation). In terms of prevention, hygiene measures are useful but are not always sufficient to prevent pseudotuberculosis. Vaccination could thus be valuable to prevent the lethality caused by this microorganism in animals living in captivity. The veterinary vaccine mainly used in European Zoos [13], named Pseudovac (Utrecht Veterinary Faculty, the Netherlands) is a killed whole-cell vaccine composed of serotype I to VI isolates. Produced for zoological gardens and wildlife parks, its use in birds [17-19] and small primates $[20,21]$ has been reported. However, its efficacy has never been really evaluated. Furthermore, pseudotuberculosis cases were observed in animals vaccinated with Pseudovac in zoos (unpublished observations from T. Petit and A. Maillot at the La Palmyre and Amnéville zoos, France, respectively).

In the present study, we examined whether Pseudovac could efficiently protect guinea pigs (which are highly susceptible to the disease) against a $Y$. pseudotuberculosis infection. Furthermore, we compared the protection conferred by Pseudovac to that of a live attenuated strain of $Y$. pseudotuberculosis (IP32680) administered orally. This strain was previously shown to be avirulent in mice [22]. We show here that Pseudovac is inefficient in protecting against a severe $Y$. pseudotuberculosis infection and that oral vaccination with IP32680 is much more protective and may represent a valuable alternative to the currently used veterinary vaccine.

1.

1.1 .

\section{Materials and Methods}

Yersinia strains and culture conditions.

The serotype II Y. pseudotuberculosis strain IP32680 was isolated from a dead hare. IP32680 lacks genetic determinants coding for known virulence factors such as the High Pathogenicity Island or the super antigens, but harbors the pYV virulence plasmid or the psa fimbriae [22]. This strain was found to be avirulent in the mouse model since oral or sc inoculation of high doses $\left(10^{9} \mathrm{cfu}\right)$ of IP32680 failed to kill or to cause detectable signs of disease in these animals. All Y. pseudotuberculosis isolates (serotypes I to V) used in this study were taken from the collection of the Yersinia Research Unit (Institut Pasteur) and were positive by PCR for the presence of the pYV virulence plasmid and the Psa fimbriae (pH6 antigen). Bacteria were 
usually grown at $28^{\circ} \mathrm{C}$ in Luria-Bertani agar plates supplemented with $0.2 \%$ hemin (LBH) for $48 \mathrm{~h}$ before use.

Female Hartley guinea pigs (six to eight weeks old) were purchased from Charles River (L'Arbresle, France). All animal care and experimentations were conducted according to the guidelines of the Institut Pasteur and Ecole Nationale Vétérinaire (France). Anesthesia was obtained by intramuscular (im) injection of Zoletil 100 (Virbac, France) or Xylazine (Rompun ${ }^{\circledR}$ from Bayer; $2.5 \mathrm{mg} / \mathrm{kg}$ ) plus Ketamine (Imalgene 1000® from Mérial; $125 \mathrm{mg} / \mathrm{kg}$ ). Bacterial suspensions (200 $\mu \mathrm{l}$ in saline) of the serotype II strain IP32680 were inoculated via the intragastric (ig) route using a curved feeding needle. To evaluate the virulence of the IP32680 strain, guinea pigs were inoculated ig with a high bacterial dose $\left(10^{9} \mathrm{cfu}\right)$ and survival was followed for 3 weeks. At the end of the experiment, animals were euthanized by intraperitoneal (ip) injection of Pentobarbital $(200 \mathrm{mg} / \mathrm{kg})$ and the presence of lesions in the intestine, mesenteric lymph nodes, liver, spleen and lungs were searched for. Peyer's patches were also examined and the number and size of enlarged patches were recorded. In order to evaluate the clearance of the vaccine bacteria from the body, various organs ( 2 swollen Peyer's patches, mesenteric lymph nodes, spleen, liver and lungs) were sterily taken, cut into pieces and minced using glass beads (VWR, USA) and an electric mill (Retsch, Germany). Bacterial counts were obtained by plating dilutions of homogenates on LB agar (Table I).

To determine the $50 \%$ lethal dose $\left(\mathrm{LD}_{50}\right)$ of strain IP32953 for guinea pigs inoculated ig, four animals per dose were infected with 10 fold serial dilutions of IP32953 and survival was followed for 3 weeks. Vaccination with the IP32680 strain consisted in one or two ig inoculation of $10^{9} \mathrm{cfu}$ in saline at 40-day interval. The Pseudovac vaccine was obtained from the Microbiology Laboratory of the Zoo and Exotic animals section of the Utrecht Veterinary Faculty. Vaccination using Pseudovac consisted in two subcutaneous (sc) injections $(0.25 \mathrm{ml}$ each) in the back of the guinea pigs at 40-day interval, as recommended for animals below one $\mathrm{kg}$ of weight. Although the name "Pseudovac" has been used for this vaccine for years, it is not a registered trademark and a distinct vaccine against Pseudomonas aeruginosa now also has this name.

In order to evaluate guinea pigs protection against pseudotuberculosis after vaccination, animals received a suspension of $10^{9} \mathrm{cfu}\left(2,200 \times \mathrm{LD}_{50}\right)$ of the virulent Y. pseudotuberculosis strain IP32953 inoculated ig 30 days after the last vaccine dose. Thereafter, animal mortality, behavior and fur appearance were recorded daily for three weeks. Body weight was measured every three - four days as a marker of health. At the end of the experiment, animals were euthanized and the presence of lesions in the intestine, mesenteric lymph nodes, liver, spleen and lungs were searched for as described above.

To obtain immune serum, guinea pigs were anesthetized and blood was collected from the retro-orbital sinus or the ear veins. Serum was frozen until use. To produce the $Y$. pseudotuberculosis antigenic solutions used for coating in the ELISA assays, bacteria were grown on LB agar for $48 \mathrm{~h}$ either at $28^{\circ} \mathrm{C}$ to allow expression of $\mathrm{O}-\mathrm{Ag}[23,24]$, or at $37^{\circ} \mathrm{C}$ to favor the expression of molecules up regulated in the host, including the Type Three Secretion System (TTSS) and the Yops effectors [25]. Bacterial antigenic preparations were produced by sonication of live bacteria and were used for coating in ELISA assays as previously described 
[22]. Sera were serially diluted in PBS containing 0.1\% BSA for determination of antibody titers by ELISA. Secondary antibodies (Ab) against guinea pig IgG coupled to horseradish peroxydase (Jackson Immunoresearch) were used, followed by colorimetric revelation using TMB (OptiEIA, BD-biosciences) as previously described [22]. Titers were calculated as the sample dilution giving an $\mathrm{OD}_{450}$ of 0.5 on the titration curve.

1.4 .

Statistical analysis

The Spearman-Karber test was used to calculate the $\mathrm{LD}_{50}$ and its confidence interval for strain IP32953 inoculated ig. Linear regression analysis was performed to evaluate the correlation between antibody titers against different serotypes of $Y$. pseudotuberculosis. The Mantel-Cox Logrank test was used to evaluate the protection of guinea pigs.

2. Results

2.1. Induction of acute pseudotuberculosis in the guinea pig

The protective value of pseudotuberculosis vaccines was evaluated in the guinea pig model because this species is highly susceptible to a $Y$. pseudotuberculosis infection [26, 27]. Moreover, since they are not inbred animals, the efficiency of vaccination may vary from animal to animal similarly to the non-laboratory animals for which the vaccine is developed. To perform a challenge infection, a model of acute and lethal pseudotuberculosis in guinea pigs was required. The lethal dose $50 \%\left(\mathrm{LD}_{50}\right)$ of the highly virulent $Y$. pseudotuberculosis strain IP32953 [28] via the ig route was determined. The $\mathrm{LD}_{50}$ observed was of $4.6 \times 10^{5} \mathrm{cfu}(95 \%$ confidence interval: $\left.2.2 \times 10^{4}-9.7 \times 10^{6} \mathrm{cfu}\right)$. Based on the $\mathrm{LD}_{50}$ and on the fact that the bacterial inoculum necessary to cause a natural infection is not known but may be high, we decided to use a dose of $10^{9} \mathrm{cfu}$ of IP32953 (corresponding to $2,200 \mathrm{LD}_{50}$ ) to mimic a severe infection. At this dose, 17 out of 18 infected animals succumbed to the infection (Table II) with a mean day to death of 7.1 \pm 2.1 days. Death was always preceded by prostration, ruffled fur and loss of appetite with severe weight loss (not detailed). Post-mortem examination of the dead guinea pigs always revealed pseudotuberculous nodular lesions visible in the intestine, caecum, spleen and liver. In $40 \%$ of animals, the lungs were also affected.

\section{Evaluation of the immune response and protection induced by Pseudovac}

Vaccination using Pseudovac as recommended by the producer consists in two sc injections at a 40 day interval. The serum of vaccinated guinea pigs was collected 30 days after the last inoculation (just before challenge) to evaluate the antibody response induced. The IgG titers against five different acellular preparations of $Y$. pseudotuberculosis strains of serotypes I to $\mathrm{V}$ were determined by ELISA. All guinea pigs having received Pseudovac mounted a significant IgG response against all five serotypes of $Y$. pseudotuberculosis (Fig. 2, $\mathrm{p}<0.005$ for all serotypes). Surprisingly, only 1 out of 12 animals vaccinated with 2 sc injections of Pseudovac survived the infection (Table II), and the kinetics of death (7.0 \pm 2.4 days) was not significantly different from that of unvaccinated animals. These results indicate that the antibody response mounted by the vaccinated animals is not protective and that Pseudovac is not efficient in protecting against a severe $Y$. pseudotuberculosis infection. 
We decided to evaluate the possibility of using the live attenuated $Y$. pseudotuberculosis strain IP32680 as an oral vaccine against animal pseudotuberculosis because this strain was previously shown to be avirulent in a mouse model of oral or subcutaneous infection and to induce a protective immunity against bubonic plague [22]. To determine whether IP32680 is also avirulent in the guinea pig model, a total of 40 animals were inoculated ig with $10^{9}$ cfu of strain IP32680. Not a single animal died or presented clinical symptoms of infection such as weight loss (Fig. 1) or ruffled fur over the follow-up period of 80 days. Post-mortem examination of three animals euthanized at day 21 post-infection showed that they all presented several enlarged Peyers' patches (average size 7-9 mm), reflecting cellular recruitment from the blood and proliferation of antigen-specific $\mathrm{T}$ and $\mathrm{B}$ cells. The Peyers' patches of two animals contained low amounts of live bacteria (Table I), thus indicating a long lasting stimulation of the intestinal immune system. In contrast, no bacteria were recovered from the mesenteric lymph nodes, spleen, liver and lungs of these animals (Table I), and no lesions were observed in these organs. In animals that received a second inoculation of $10^{9} \mathrm{cfu}$ of IP32680 to restimulate their immune response 40 days after the first inoculation, no sign of disease was noted (data not shown). Therefore, we concluded that IP32680 is avirulent for guinea pigs.

To evaluate the ability of IP32680 to confer protection against pseudotuberculosis, two vaccination protocols were tested, consisting in either one or two oral inoculations (prime / boost strategy) of IP32680 ( $\left.10^{9} \mathrm{cfu}\right)$ at a 40 day interval. The antibody response directed against whole bacterial antigens was evaluated 30 days after the last dose, just prior to challenge. Animals which had received one ig inoculation of IP32680 (serotype II) not only mounted a significant IgG response against serotype II, but also against the other serotypes (I to V) of $Y$. pseudotuberculosis grown at $28^{\circ} \mathrm{C}$ to allow in vitro expression of $\mathrm{O}$-antigens (Figure $2 ; \mathrm{p}$ at least $<0.005$ for all serotypes). Linear regression analysis showed a strong correlation (slope each time close to $1, \mathrm{p} \leq 0.001$ ) between the titers induced by IP32680 against serotype II and the other serotypes (Fig. 3). This shows that antibodies against serotype II O-antigens represent a minor part of the bacteria-specific antibodies. These IgG titers after one inoculation of IP32680 were comparable to those induced by the two sc injections of Pseudovac (Fig. 2). In guinea pigs having received two ig inoculations of IP32680, a two to four fold increase of the IgG titers against all five serotypes of $Y$. pseudotuberculosis was observed (Fig. 2). This increase was statistically significant $(p \leq 0.005)$ for serotypes I, II and III strains, showing that this second inoculation boosted the immune response. IgG titers were also determined with antigenic preparations of bacteria grown at $37^{\circ} \mathrm{C}$, a temperature closer to in vivo conditions and which allows expression of virulence factors such as the pYV-encoded Type III Secretion System and Yops. The IgG titers were comparable to those observed with bacteria grown at $28^{\circ} \mathrm{C}$ (data not shown), indicating that target antigens expressed at the host's body temperature are also recognized by IP32680-induced IgG.

Whereas control unvaccinated animals died of infection (paragraph 3.1) one oral inoculation of IP32680 provided protection $(\mathrm{p}=0.01)$ to $6 / 12$ animals (Table II) against an otherwise lethal challenge with $10^{9} \mathrm{cfu}$ of IP32953, indicating that IP32680 induces a higher protection than Pseudovac. In the second group which received two oral inoculations of IP32680, $5 / 6$ animals survived the challenge $(\mathrm{p}=0.01$, Table II), showing that this prime / boost vaccinal 
strategy further increased the protection conferred by IP32680. Animals protected by two inoculations of IP32680 were euthanized at day 21 post challenge and necropsy revealed no lesions in visceral organs, except for one animal which had a small nodule in the liver. In each of these protected animals, two to five hypertrophied Peyers' patches $(7-10 \mathrm{~mm})$ were visible on the intestine, as also observed prior to challenge in IP32680-vaccinated animals.

2.6. Correlation between antibody titers against IP32680 and protection against pseudotuberculosis.

To evaluate the value of Yersinia-specific IgG titers measured before challenge as predictors of protective immunity, the titers obtained in the six guinea pigs protected by one inoculation of IP32680 were compared to those of the six unprotected animals. Although protected animals tended to develop higher IgG titers than unprotected ones (mean 49,978 versus 39,860 respectively), antibody titers were not statistically different in the two groups, showing that they are not accurate indicators of protection.

3.

\section{Discussion}

The only vaccine presently in use in Europe to vaccinate zoo and wildlife park animals against pseudotuberculosis is Pseudovac. This vaccine is composed of formalin-killed $Y$. pseudotuberculosis isolates of serotypes I to VI, and its manufactor recommends to perform two sc injections every year. We evaluated the efficacy of Pseudovac to induce an immune response against $Y$. pseudotuberculosis and to protect guinea pigs against acute pseudotuberculosis caused by ingestion of the highly virulent strain IP32953. Surprisingly, whereas Pseudovac is able to induce a strong humoral immune response against $Y$. pseudotuberculosis antigens in vaccinated animals, it fails to confer them any protection against the disease. Our results contrast with previous reports indicating some protective effect of Pseudovac in birds and small primates in zoos [17, 18, 21, 19], but are in agreement with our observation of pseudotuberculosis infections occurring in birds and primates vaccinated with Pseudovac in a zoo. The lack of activity of Pseudovac in our model could be at least partly attributable to the fact that we used high doses of the highly virulent $Y$. pseudotuberculosis strain IP32953 as challenge. If so, this would suggest that Pseudovac protective effect might be limited to mild $Y$. pseudotuberculosis infections.

As an alternative, we tested the possibility of vaccinating animals orally against pseudotuberculosis with an attenuated $Y$. pseudotuberculosis strain. It was previously shown that Y. pseudotuberculosis strain IP32680 is not virulent in the mouse model of oral or sc infection. In the present work, we found that IP32680 is also avirulent for highly sensitive animals such as guinea pigs since oral administration of high bacterial inocula $\left(10^{9} \mathrm{cfu}\right.$, corresponding to $>2,000$ times the $\mathrm{LD}_{50}$ of the virulent strain IP32953) was unable to kill any animal, to cause clinical symptoms or to induce histological lesions in deep organs.

In contrast, the detection of live bacteria only in the Peyer's patches of the animals one month after the infection indicated a colonization of the intestinal tract, thus favoring a longlasting stimulation of the immune system. Indeed, oral inoculation of $Y$. pseudotuberculosis IP32680 to guinea pigs once or twice induced a significant protection $(50 \%$ and $83 \%$ respectively) against a challenge with a high inoculum $\left(10^{9} \mathrm{cfu}\right)$ of the highly virulent $Y$. pseudotuberculosis strain IP32953. This demonstrates that the immune response elicited by live $Y$. pseudotuberculosis cells is much more efficient than that induced by the killed bacteria present in Pseudovac. In a previous study, it was observed that mice receiving an oral live avirulent TTSS mutant of $Y$. pseudotuberculosis were protected against oral pseudotuberculosis 
[29]. A dam mutant of strain IP32953 administered orally has also been shown to protect against an intravenous challenge with the IP32953 strain [30]. In both studies, the mice were challenged with a dose corresponding to $100 \mathrm{LD}_{50}$. This is in accordance with our preliminary experiments performed in the mouse model, in which we found that after a single oral inoculation with IP32680, OF1 animals were $100 \%$ protected against an oral infection with $10^{9} \mathrm{cfu}\left(50 \mathrm{LD}_{50}\right)$ of IP32953 (unpublished results). Guinea pigs are more susceptible than age-matched OF1 mice to an oral infection with the virulent IP32953 strain (LD L $_{50}$ ig of $4.6 \times 10^{5} \mathrm{cfu}$, versus $1.6 \times 10^{7} \mathrm{cfu}$ respectively [22]). This study thus shows that IP32680 has a protective effect, even against a highly severe infection $\left(2,200 \mathrm{LD}_{50}\right)$.

One oral dose of the IP32680 strain induced a humoral response close to what was obtained with two sc injections of Pseudovac, showing that the live vaccine stimulates the immune system more strongly than the vaccine made of killed bacteria. It also appeared that the IgG produced against IP32680 (serotype II) were able to recognize strains of other serotypes. The classification of $Y$. pseudotuberculosis strains in serotypes is based on differences in the carbohydrate branching (O-Antigen) of the conserved core of LPS, the main component of the outer membrane of Gram negative bacteria [31]. Our observations indicate that the immune response elicited by IP32680 is directed against antigens common to all $Y$. pseudotuberculosis strains, whatever their serotype, and that the mix of different serotypes in a vaccine is not necessary. Indeed, vaccination with IP32680 (serotype II) protected against infection with the IP32953 strain (serotype I).

A whole-cell vaccine like IP32680 induces an immune response directed against many antigens including surface exposed structures ( $\mathrm{O}$-antigen, outer membrane proteins, fimbriae) and virulence factors (Yops, etc.). Antibodies are important players of the anti-bacterial immunity due to their capacity to facilitate the phagocytosis of antibody-covered ("opsonized") bacteria, and by blocking the function of surface molecules important for the virulence. However, two observations suggest that a strong humoral immune response is not sufficient to predict protection against pseudotuberculosis: first, the high IgG titers induced by Pseudovac were not protective, and second, in animals vaccinated with one injection of IP32680, IgG titers of surviving guinea pigs were not statistically different from those of the animals who died. Nonetheless, although IgG titers do not predict protection, it cannot be excluded that some components of the humoral response may participate to the protective effect observed. Protective antibodies may be able to work in association with the cellular response, and to neutralize virulence factors like Yops [32], and new tests based on these properties may be more efficient in predicting protection. Following this view, the abundant antibodies induced by Pseudovac could be unefficient because they are directed against formalin-treated antigens and so would fail to neutralize native antigens.

Live vaccines present several advantages: (i) they include multiple antigens that are produced de novo as long as the bacteria persist in the host, thus providing a prolonged stimulation of the immune system. (ii) live organisms regulate their gene expression to adapt to in vivo conditions, and thus expose target antigens that might not be produced during growth in vitro, (iii) they also generally induce both an antibody and a cell-mediated response, both contributing to the vaccination efficiency [33]. As compared to molecular vaccines, whole-cell vaccines also allow low cost mass production. Furthermore, oral vaccination, in contrast to parenteral injection, targets the development of mucosal immunity in the gut, the first site of $Y$. pseudotuberculosis infection, as confirmed by the observation of enlarged Peyer's patches in the intestine. We previously reported that $Y$. pseudotuberculosis strain IP32680 given orally to mice persist in the gut for up to two months, exerting a prolonged stimulation of the immune system 
[22]. The oral route was also the rationale for the recent use of Lactococcus lactis expressing the $Y$. pseudotuberculosis LcrV antigen as a candidate vaccine against pseudotuberculosis [34]. Oral vaccines are also easier to administer, and less stressful because they can be included in food so no animal capture is necessary. Immunization using a vaccine hidden in food baits may also be applied to wild animals, a strategy successfully used to vaccinate foxes against rabies [35]. This strategy could be used to treat reservoir animals in the proximity of farms, zoos or wildlife parks.

\section{Conclusion}

The present work shows that, in contrast to the vaccine Pseudovac currently in use for veterinary purposes, a live attenuated strain of $Y$. pseudotuberculosis given orally confers protection to guinea pigs against an acute and severe infection. The IP32680 strain administered orally thus represents a valuable alternative vaccine to Pseudovac, usable in zoos, wildlife parks and livestock, where precious animals are at high risk of pseudotuberculosis.

\section{Acknowledgements}

B. Quintard was supported by the Association Française des Vétérinaires de Parcs Zoologiques and the Virbac Company. The authors wish to thank the French Zoos who supported this work.

\section{Conflict of Interest}

The authors have no conflicts of interest. 


\section{References}

1. Fukushima $\mathrm{H}, Y$. enterocolitica and $Y$. pseudotuberculosis: Epidemiology of $Y$. pseudotuberculosis. In: Dworkin W, Falkow S, Rosenberg E, Schleifer K-H,Stackebrandt E, editor. The Prokaryotes, a Handbook on the Biology of Bacteria. New York: Springer-Verlag, 2006. p. 15-20.

2. Inoue M, Nakashima H, Ueba O, Ishida T, Date H, Kobashi S, et al. Community outbreak of Yersinia pseudotuberculosis. Microbiology and Immunology 1984; 28(8):883-891.

3. Jalava K, Hallanvuo S, Nakari UM, Ruutu P, Kela E, Heinasmaki T, et al. Multiple outbreaks of Yersinia pseudotuberculosis infections in Finland. Journal of Clinical Microbiology 2004; 42(6):2789-2791.

4. Somov GP, Martinevsky IL. New Facts about Pseudotuberculosis in the USSR. Contributions to Microbiology and Immunology 1973; 2(214-216.

5. Fukushima H, Gomyoda M. Intestinal Carriage of Yersinia pseudotuberculosis by Wild Birds and Mammals in Japan. Applied and Environnemental Microbiology 1991; 57(4):1152-1155.

6. Callinan RB, Cook RW, Boulton JG, Fraser GC, Unger DB. Enterocolitis in cattle associated with Yersinia pseudotuberculosis infection. Australian Veterinary Journal 1988; 65(1):8-11.

7. Riet-Correa F, Gil-Turnes C, Reyes JC, Schild AL, Mendez MC. Yersinia pseudotuberculosis infection of buffaloes (Bubalus bubalis) Journal of Veterinary Diagnostic Investigation 1990; 2(1):78-79.

8. Sanford SE. Outbreaks of yersiniosis caused by Yersinia pseudotuberculosis in farmed cervids. Journal of Veterinary Diagnostic Investigation 1995; 7(1):78-81.

9. Slee KJ, Skilbeck NW. Epidemiology of Yersinia pseudotuberculosis and Yersinia enterocolitica Infections in Sheep in Australia. Journal of Clinical Microbiology 1992; 30(3):712-715.

10. Baskin GB, Montali RJ, Bush M, Quan TJ, Smith E. Yersiniosis in captive exotic mammals. Journal of the American Veterinary Medical Association 1977; 171(9):908-912.

11. Kageruka P, Mortelmans J, Vercruysse J, Beernaert-Declercq C. Pseudotuberculosis in the Antwerp Zoo. Acta Zool. Pathol. Antverp. 1976; 66):111-120.

12. Parsons R. Pseudotuberculosis at the Zoological Society of London (1981 to 1987). The Veterinaary Record 1991; 128(6):130-132.

13. Williams C, Approaches to control Yersinia pseudotuberculosis in European Zoos. In: EAZWV, editor. European Association of Zoo- and Wildlife Veterinarians (EAZWV) 5th scientific meeting. Ebeltoft. Denmark, 2004. p. 19-25.

14. Welsh RD, Ely RW, Holland RJ. Epizootic of Yersinia pseudotuberculosis in a wildlife park. Journal of the American Veterinary Medical Association 1992; 201(1):142-144.

15. Vincent P, Leclercq A, Martin L, Duez JM, Simonet M, Carniel E. Sudden onset of pseudotuberculosis in humans, France, 2004-05. Emerg Infect Dis 2008; 14(7):1119-1122.

16. Gurleva GG, Korganov Ia N, Makarovskaia LN, Zosimenko VS, Popov VF. [Antibiotic sensitivity of Yersinia enterocolitica and Yersinia pseudotuberculosis isolated from patients with the jaundice form of acute hepatitis]. Antibiotiki 1982; 27(1):37-41.

17. Borst GH, Buiterlaar M, Poelma FG, Zwart P, Dorrestein GM. Yersinia pseudotuberculosis in birds. Tijdschr Diergeneeskd 1977; 102(2):81-85.

18. Dorrestein GM, Zwart P, Borst GH, Poelma FG, Buitelaar MN. Disease and causes of death in birds. Tijdschr Diergeneeskd 1977; 102(7):437-447. 
19. Zwart P, Wiesner H, Göltenboth R, Erfahrungen mit dem einsatz einer pseudotuberkulosetotvakzine bei vögeln, XXIII. Internationalen Symposiums über die Erkrankugen der Zootiere. (1981) 73-76.

20. Bakker J, Kondova I, de Groot C, Remarque E, Heidt P. A report on Yersinia-related mortality in a colony of new world monkeys. Laboratory Primate Newsletter 2007; 46(3):11-15.

21. Poelma F, de Voogt G, Immunisierung von Affen gegen Pasteurella pseudotuberculosis mit einer Totvakzine aus virulenten Bakterienstämme., XI International Symposium Erkrankungen der Zootiere. (1969) 89-91.

22. Blisnick T, Ave P, Huerre M, Carniel E, Demeure CE. Oral vaccination against bubonic plague using a live avirulent Yersinia pseudotuberculosis. Infect Immun 2008; 76(8):3808-3816.

23. Bengoechea JA, Brandenburg K, Seydel U, Diaz R, Moriyon I. Yersinia pseudotuberculosis and Yersinia pestis show increased outer membrane permeability to hydrophobic agents which correlates with lipopolysaccharide Acyl-chain fluidity. Microbiology 1998; 144(Part 6):15171526.

24. Pouillot F, Derbise A, Kukkonen M, Foulon J, Korhonen TK, Carniel E. Evaluation of Oantigen inactivation on Pla activity and virulence of Yersinia pseudotuberculosis harbouring the pPla plasmid. Microbiology 2005; 151(Pt 11):3759-3768.

25. Cornelis GR. The Yersinia YSC-YOP 'type III' weaponry. Nature Reviews Molecular Cell Biology 2002; 3(10):742-752.

26. Rigby C. Natural infections of guinea pigs. Laboratory Animals 1976; 10(2):119-142.

27. Wagner JE, Manning PJ. The Biology of the Guinea Pig, 3rd ed ${ }^{\wedge}$. NY: Academic Press, 1976.

28. Chain PS, Carniel E, Larimer FW, Lamerdin J, Stoutland PO, Regala WM, et al. Insights into the evolution of Yersinia pestis through whole-genome comparison with Yersinia pseudotuberculosis. Procedings of the National Academy of Sciences of the United States of America 2004; 101(38):13826-13831.

29. Balada-Llasat JM, Panilaitis B, Kaplan D, Mecsas J. Oral inoculation with Type III secretion mutants of Yersinia pseudotuberculosis provides protection from oral, intraperitoneal, or intranasal challenge with virulent Yersinia. Vaccine 2007; 25(8):1526-1533.

30. Taylor VL, Titball RW, Oyston PCF. Oral immunization with a dam mutant of Yersinia pseudotuberculosis protects against plague. Microbiology 2005; 151(1919-1926.

31. Tsubokura M, Aleksic S. A simplified antigenic scheme for serotyping of Yersinia pseudotuberculosis: phenotypic characterization of reference strains and preparation of $\mathrm{O}$ and $\mathrm{H}$ factor sera. Contributions to Microbiology and Immunology 1995; 13(99-105.

32. Welkos S, Norris S, Adamovicz J. Modified caspase-3 assay indicates correlation of caspase3 activity with immunity of nonhuman primates to Yersinia pestis infection. Clin. Vaccine Immunol. 2008; 15(7):1134-1137.

33. Smiley ST. Current challenges in the development of vaccines for pneumonic plague. Expert Review of Vaccines 2008; 7(2):209-221.

34. Daniel C, Sebbane F, Poiret S, Goudercourt D, Dewulf J, Mullet C, et al. Protection against Yersinia pseudotuberculosis infection conferred by a Lactococcus lactis mucosal delivery vector secreting LcrV. Vaccine 2009; 27(8):1141-1144.

35. Pastoret PP, Brochier B. Epidemiology and control of fox rabies in Europe. Vaccine 1999; 17(13-14):1750-1754. 


\section{Figure legends}

Figure 1: Guinea pigs do not lose weight after vaccination with $Y$. pseudotuberculosis IP32680 inoculated ig or Pseudovac injected sc.

Guinea pigs received either the Pseudovac vaccine $(0.25 \mathrm{ml} \mathrm{sc})$ at days 0 and 40 (indicated by arrows), saline sc (control group) at the same days, or a single inoculation (109 $\mathrm{cfu})$ of strain IP32680 ig at day 40, and their individual weight was measured at regular intervals. Shown are means \pm SEM of 20 guinea pigs per group.

Figure 2: Vaccination sc with the killed whole-cell vaccine Pseudovac or ig with the live IP32680 strain induce a strong IgG response against $Y$. pseudotuberculosis antigens.

Serum samples were obtained from groups of guinea pigs that had received either $10^{9} \mathrm{cfu}$ of IP32680 ig once $(n=20)$ or twice $(n=6)$, or Pseudovac injected sc twice $(n=20)$, or saline (control group, $\mathrm{n}=20$ ) sc twice, at a 40 day interval. Blood samples were collected 30 days after each vaccine or saline dose and serum IgG titers against $Y$. pseudotuberculosis antigens were determined using ELISA plates coated with filtered sonicates of $Y$. pseudotuberculosis strains serotype I to $\mathrm{V}$. The results (mean IgG titers \pm SEM) have been obtained with preparations of bacteria grown at $28^{\circ} \mathrm{C}$ to favor expression of $\mathrm{O}$-antigens. Equivalent results were obtained with bacteria grown at $37^{\circ} \mathrm{C}$. Not shown with asterisks in the figure is the fact that all 3 vaccination protocols induce a strongly significant increase in IgG titers (all at least $p<0.005$ ). ${ }^{*}$ : $p<0.05$

Figure 3: Guinea pig serum IgG induced by oral vaccination with strain IP32680 (serotype II) are reactive against other serotypes of $Y$. pseudotuberculosis

Blood samples were collected 30 days after oral inoculation of $10^{9} \mathrm{cfu}$ of IP32680. ELISA were then performed as in Fig. 2. Shown are individual titers from 32 guinea pigs pooled from three independant experiments. Equivalent results were obtained with sonicates of bacteria grown at $37^{\circ} \mathrm{C}$ to favor Yop production. The $r^{2}$ value and $p$ value obtained using Spearman's rank test are given. 


\section{Table I}

Clearance of the $Y$. pseudotuberculosis strain IP32680 after oral vaccination ${ }^{\text {a }}$

\begin{tabular}{cccccc}
\hline & \multicolumn{5}{c}{ Y.pseudotuberculosis counts ${ }^{\mathrm{b}}$} \\
\cline { 2 - 6 } Guinea pig & Peyer's patches & Mesenteric LN & Liver & Lungs & Spleen \\
\hline 1 & 70 & 0 & 0 & 0 & 0 \\
2 & 93 & 0 & 0 & 0 & 0 \\
3 & 0 & 0 & 0 & 0 & 0 \\
\hline
\end{tabular}

(a) Guinea pigs were orally inoculated with $10^{9}$ cfu of $Y$. pseudotuberculosis strain IP32680 and were sacrificed 21 days later.

(b) Organs were sterily minced and bacterial counts were obtained by plating dilutions of homogenates on LB agar. Due to the size of these organs, $1 / 10^{\text {th }}$ of liver and lungs or $1 / 5^{\text {th }}$ of spleen homogenates was plated.

\section{Table II}

Protection against an infection with the highly virulent $Y$. pseudotuberculosis strain IP32953 after vaccination by oral inoculation with IP32680 or sc injections of Pseudovac

\begin{tabular}{ccccc}
\hline Immunization & Vaccinal dose & $\mathrm{n}$ of doses & Route & Survival ${ }^{\mathrm{a}, \mathrm{b}}$ \\
\hline Saline & $0.25 \mathrm{ml}$ & 2 & ig & $1 / 18(5 \%)$ \\
Pseudovac & $0.25 \mathrm{ml}$ & 2 & $\mathrm{sc}$ & $1 / 12(8 \%)$ \\
IP32680 & $10^{9} \mathrm{cfu}$ & 1 & ig & $6 / 12(50 \%) *$ \\
IP32680 & $10^{9} \mathrm{cfu}$ & 2 & ig & $5 / 6(83 \%) * *$ \\
\hline
\end{tabular}

(a) Animals were challenged sc at day 70 with $10^{9}$ cfu of $Y$. pseudotuberculosis IP32953

(b) Results pooled from two to three experiments

$* / * *$ : Logrank test compared to saline; *: $\mathrm{p}<0.05, * *$ : $\mathrm{p}<0.01$ 


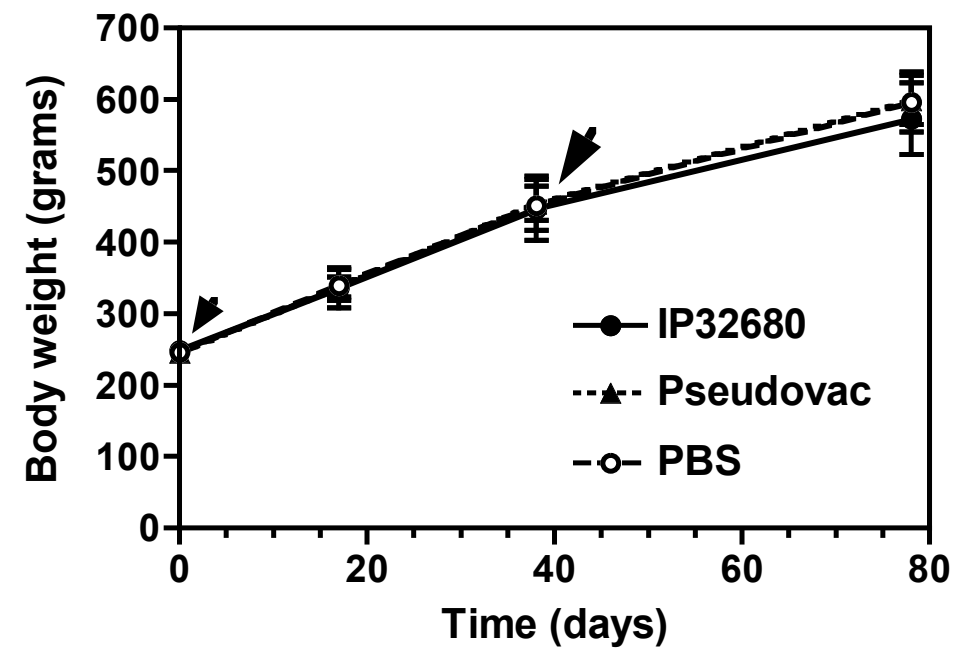


Figure 2

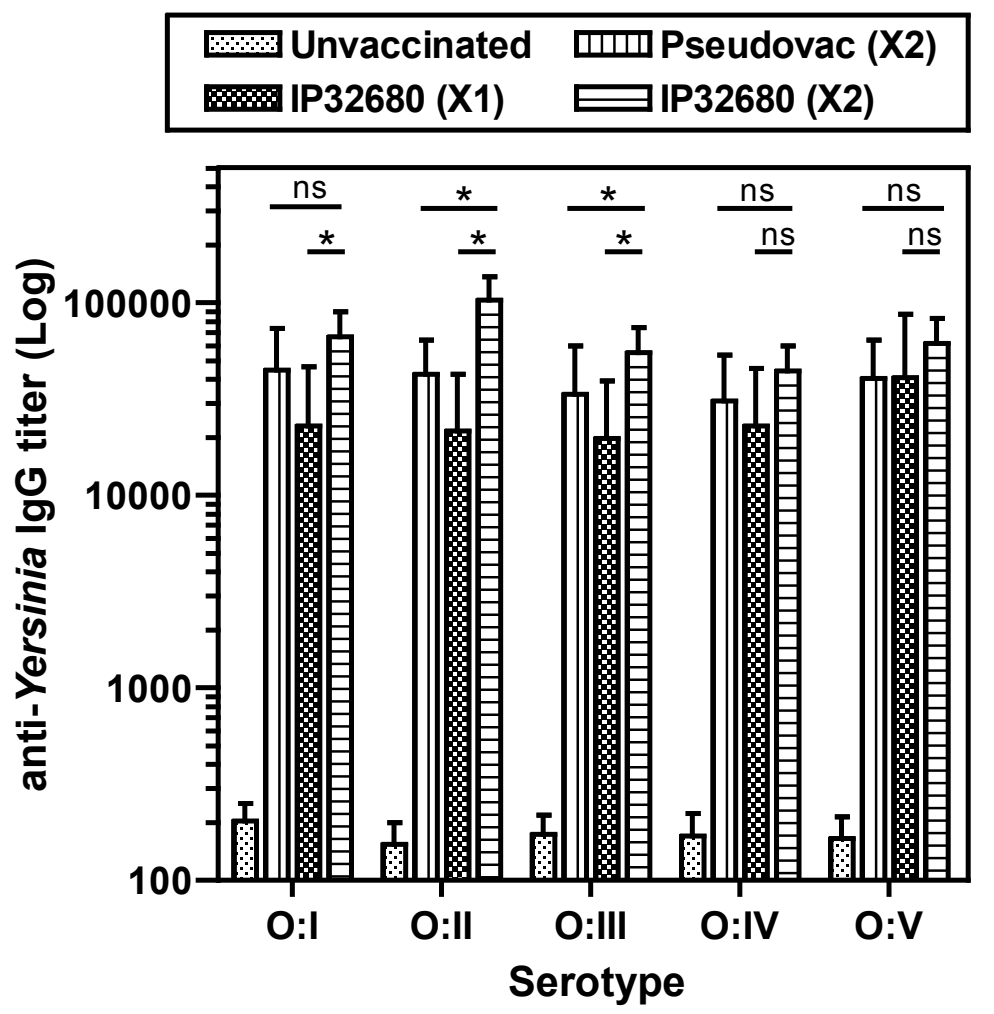


Figure 3
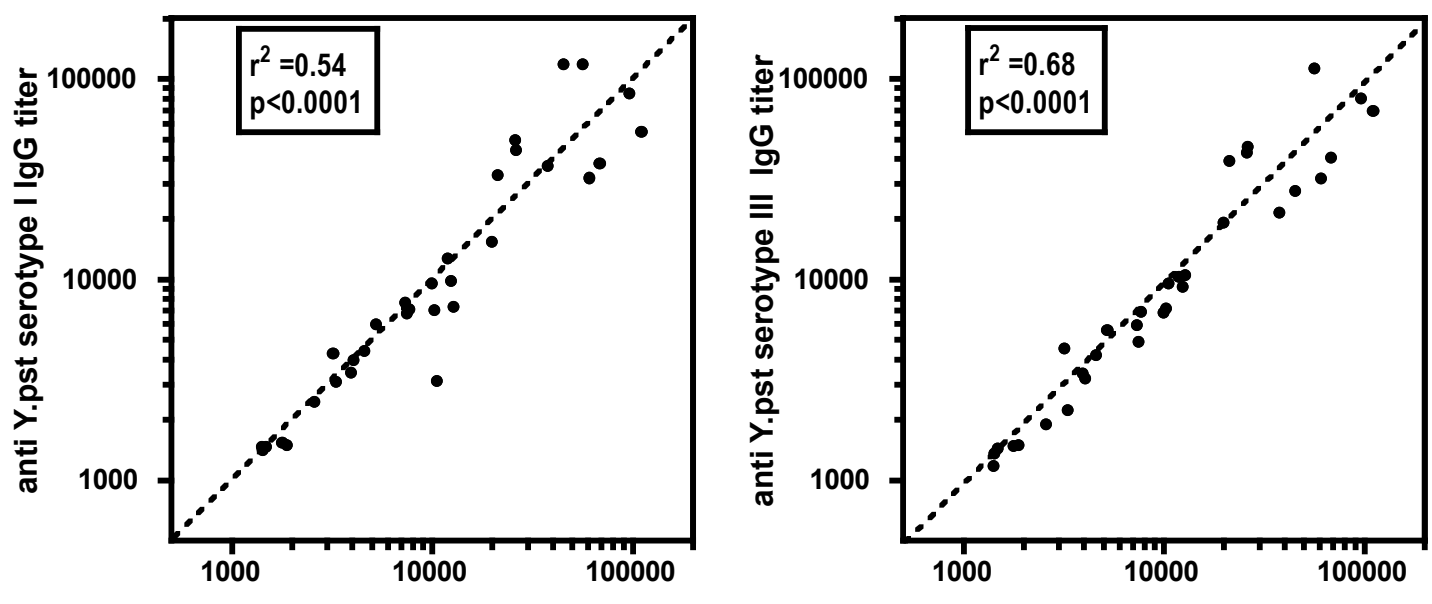

anti Y.pst serotype II IgG titer
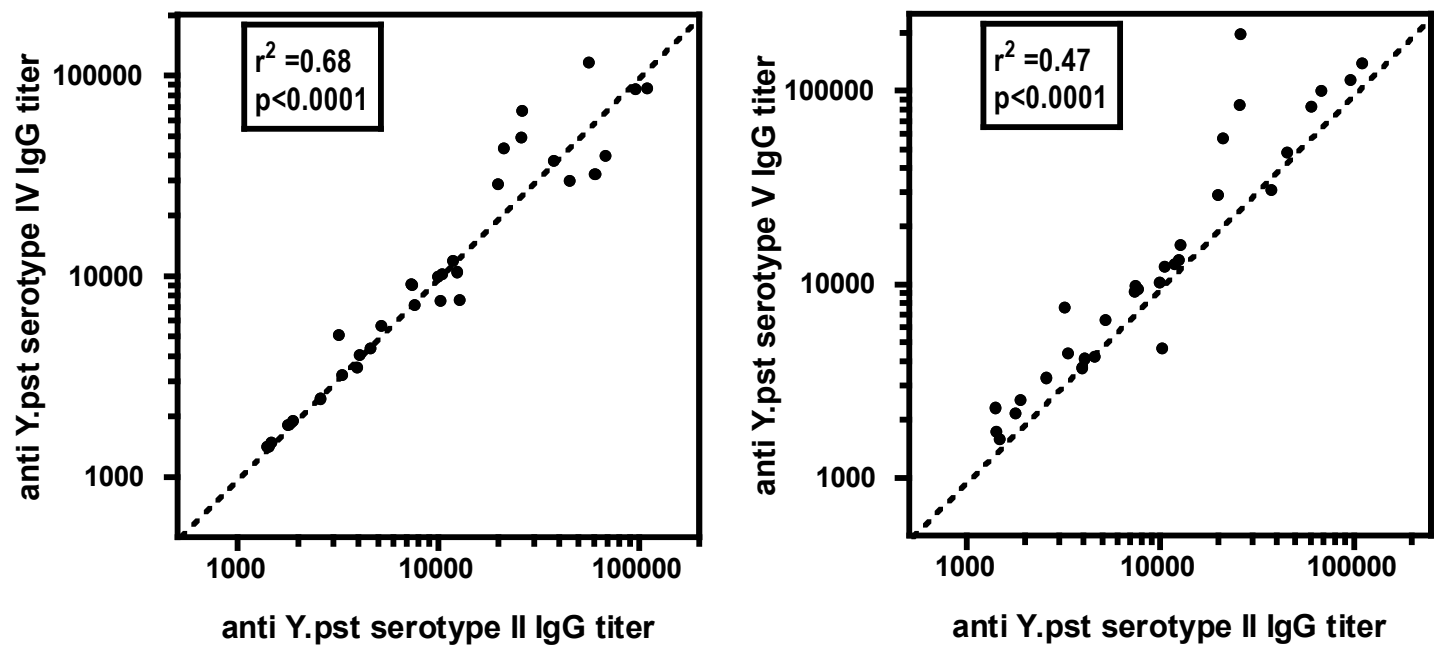
Figure 4

A

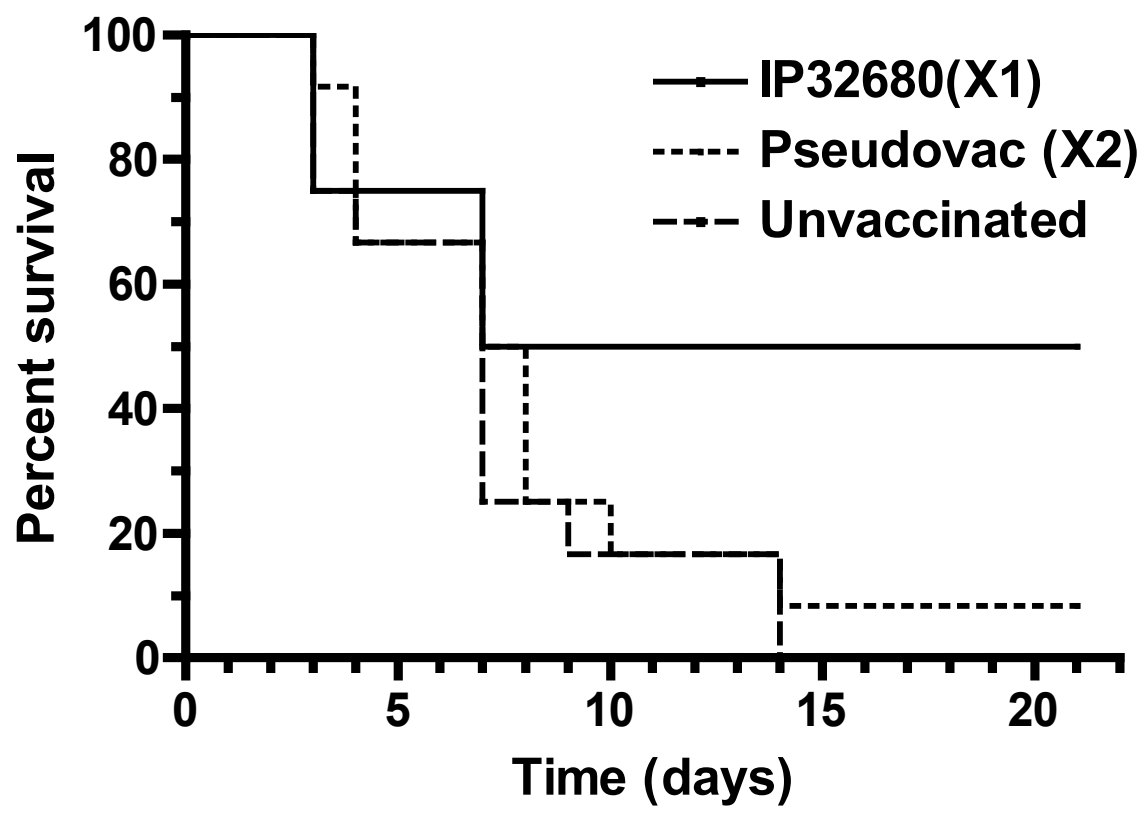

B

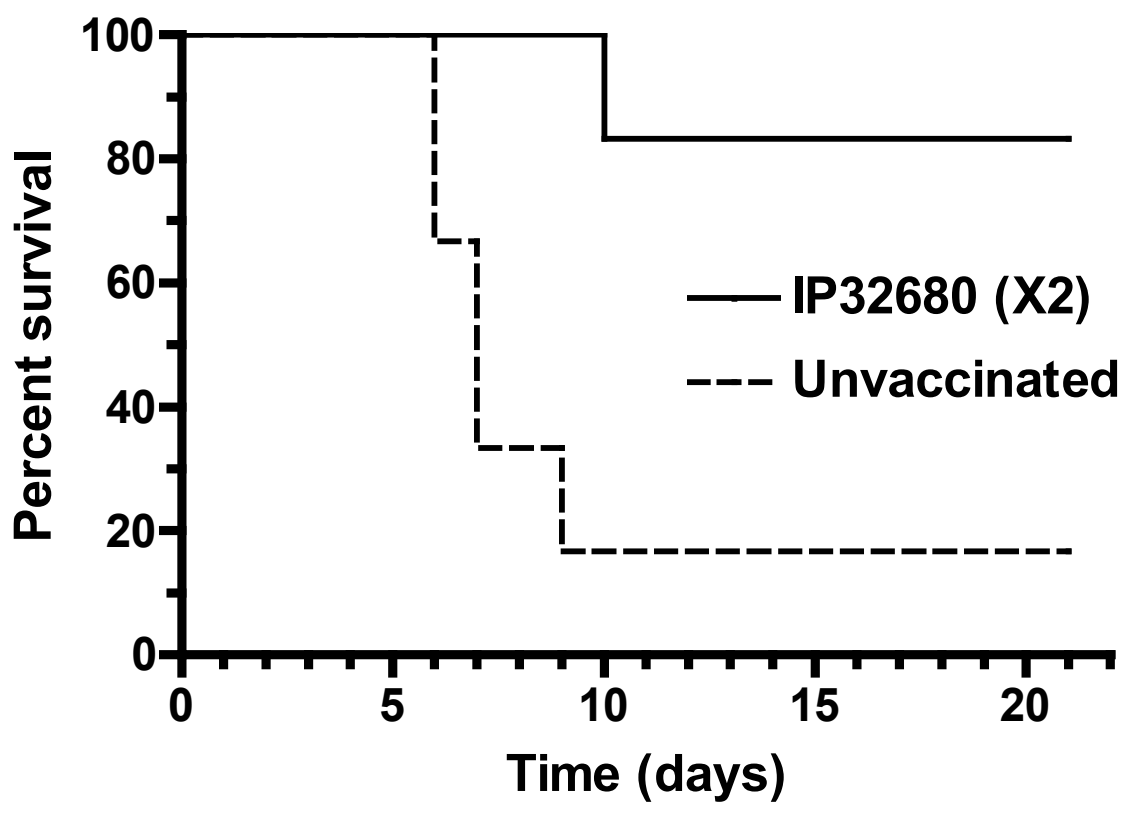

